\title{
Estrategias de aprendizaje en una interfaz gamificada Indoamérica 2018
}

\section{Learning strategies in a gamification interface Indoamerica 2018}

\author{
Rosa Elvira Ramírez Naranjo ${ }^{1}$ \\ https://orcid.org/0000-0002-4251-3248 \\ Universidad tecnológica Indoamérica, Ecuador \\ María de los Ángeles Mayorga Álvarez ${ }^{2}$ \\ https://orcid.org/0000-0001-7419-4520 \\ Patricio Lara ${ }^{3}$ \\ https://orcid.org/0000-0002-1062-5077 \\ Universidad de Granada, , Ecuador
}

Empresa de consultoría, capacitación y asesoría en investigación (EMCASIN), Ecuador

Recibido: 01-08-2019

Aceptado: 11-12-2019

\section{Cita Recomendada}

Ramírez, R., Mayorga, A., \& Lara, P., (2019). Estrategias de aprendizaje en una interfaz gamificada Indoamérica 2018. Hamut'ay, 6(3), 62-80. http://dx.doi.org/10.21503/hamu.v6i3.1847

\section{RESUMEN}

Gamificar un entorno educativo significa aprovechar los aspectos del juego para involucrar a los dicentes en una dinámica diferente en su aprendizaje. El presente estudio implementó una aplicación web basada en la metodología de la gamificación como estrategia potenciadora en el uso de estrategias de aprendizaje en la asignatura de psicología social; previo al uso de la misma se buscó determinar el tipo de estrategias de aprendizaje utilizadas por los estudiantes. La metodología que se implementó es un estudio longitudinal con un diseño cuasi experimental, Ya que se buscaba establecer si el uso de la aplicación web gamificada incidía en la utilización de estrategias de aprendizaje; las cuales fueron evaluadas a través de la escala CEVEAPEU diseñada por Gargallo; la población objeto de estudio fue 47 estudiantes de la asignatura de psicología social en la carrera de Psicología de la Universidad Tecnológica Indoamérica, la edad de los participantes es de 18 a 24 ańos entre hombre y mujeres. Los resultados del análisis estadístico con la prueba de Pearson indican una relación lineal positiva entre las estrategias motivacionales $51.8 \%$ afectivas $47 \%$ respecto al uso del interfaz gamificada. El valor de significancia demuestra que se tiene un valor menor a 0.05 por lo que se aprueba la hipótesis planteada La Prueba t de Student indicó una diferencia muy significativa ( $\mathrm{p}=$ 0.000 ), entre quienes utilizaron gamificación y quienes no lo hicieron, concluyéndose que el uso de la gamificación influye significativamente en las estrategias de aprendizaje afectivas en los estudiantes

\footnotetext{
1 Magister en Psicología Educativa y Psicólogo Clínico especializado en Psicología Educativa, por la Universidad Técnica de Ambato Tungurahua (UTA), Ecuador. Se desempeña como docente investigador en la Universidad Tecnológica Indoamérica (UTI). dra.elviraramirez. fcs@gmail.com

2 PhD. en Ciencias de la Educación, Universidad Matanza, Cuba. Especialista en Gerencia de Procesos, Maestría en Tecnologías de Información y Multimedia Educativa Universidad UNIANDES. Se desempeña como gerente investigador de EMCASIN mayorga_maria@yahoo.es 3 Magister y Especialista en Tecnología Informática Aplicada en Redes por la Universidad Tecnológica Indoamérica de Ambato Tungurahua (UTI), Ecuador. Se desempeña como docente investigador y jefe del área de Tecnología e Informática en la Universidad Tecnológica Indoamérica. patolara@uti.edu.ec
} 
de psicología de la Universidad Tecnológica Indoamérica

Palabras Clave: Estrategia de aprendizaje, Gamificación, juego, motivación.

\begin{abstract}
Gamifying an educational environment means taking advantage of game features to involve students in a different dynamics in their learning process. The present study implemented a web application based on the gamification methodology as an empowering strategy in the use of learning strategies in the subject of social psychology. Before using it, we tried to determine the type of learning strategies used by the students. With regard to the methodology, it is a longitudinal study with a quasi-experimental design. Since its aim was to establish whether the use of the gamified web application affected the use of learning strategies; which were evaluated through the CEVEAPEU scale designed by Gargallo; the target population of the study consisted of 47 students belonging to the subject of social psychology from the Psychology Degree Program of the Universidad Tecnológica Indoamérica. The age of the participants range between 18 to 24 years, among men and women. The results of the statistical analysis with the Pearson test indicate a positive linear relationship between the motivational strategies $51.8 \%$ affective $47 \%$ regarding the use of the gamification interface. The significance value shows that, there is a value of less than 0.05 , consequently the hypothesis is approved. The Student's t-test indicated a very significant difference $(p=0.000)$, between those who used gamification and those who did not, concluding that the use of gamification significantly influences affective learning strategies in psychology students of the Universidad Technological Indoamérica.
\end{abstract}

Keywords: Learning strategy, Gamification, game, motivation.

\section{INTRODUCCIÓN}

El aprendizaje presenta actualmente una dinámica diferente, el mismo está mediado por el uso de dispositivos tecnológicos, las posibilidades de auto instruirse son infinitas. Una de las perspectivas mediadoras en este proceso es la gamificación un criterio adoptado en varios ámbitos: de tipo laboral, social y educativo; al respecto son varios los aportes realizados en Europa, Asia y en América. La base de la misma se asienta en el juego interactivo, el cual condiciona a sus usuarios a través de bonificaciones. Según Teixes, 2015 el juego propicia la motivación e incide en el comportamiento de los individuos. Por lo expuesto los docentes debemos innovar la forma de abordar el conocimiento a través de nuevas propuestas como la gamificacion.
El acceso a la tecnología en el Ecuador, según la Encuesta Nacional de Ciencia Tecnología e Innovación (ACTI) desarrollada por el Instituto Nacional de Estadísticas y Censos (INEC, 2015) indica que el grupo etario con mayor influencia son sujetos entre 16 a 24 ańos, que representa el $76.1 \%$, seguido de los comprendidos entre 5 a 15 años con $59.9 \%$ (p.11). Los recursos educativos por lo tanto deben aprovechar las ventajas que ofrece la tecnología, lamentablemente una buena parte de los docentes bloquean su uso en el proceso enseńanza y aprendizaje. Bajo este contexto esta investigación tiene como finalidad Determinar la influencia de la gamificación sobre las estrategias de aprendizaje en los estudiantes de la Universidad Tecnológica Indoamérica (UTI), bajo la implementación de una interfaz gamificada en la asignatura de psicología social. 
La estrategia de aprendizaje implica una serie de procesos que el estudiante debe implementar al abordar un tema de estudio, la presente investigación asume la postura de Gargallo (2012) quien las define como aspectos conscientes que sumen el estudiante con el fin de alcanzar un aprendizaje en el cual integra elementos meta cognitivos, motivacionales, afectivos y de apoyo. El dicente que aprende sobre el uso de estas estrategias también aprende recursos metacognitivos para asimilar la información con eficacia y eficiencia. Utilizar estrategias metodológicas basadas en la perspectiva de gamificación donde se involucre el uso de las TIC en el aprendizaje, permite un desafío creativo tanto para el estudiante como para el docente, sus roles rompen esquemas el trabajo rutinario del aula, la docencia hacia universitarios quienes representan el mayor grupo de cirbernautas presenta pocos espacios de cambio de parte de sus docentes. La universidad Tecnológica Indoamérica (UTI), es una institución particular que funciona en las ciudades de Ambato y Quito de Ecuador, su finalidad es formar profesionales de calidad, cuenta con varias carreras en diferentes áreas del conocimiento entre las que se encuentra Psicología, el futuro psicólogo de la UTI debe desarrollar habilidades y destrezas que le permitan el desarrollo de un pensamiento crítico, el procesamiento de información de forma inductiva-deductiva y la autovaloración de su propio conocimiento a través de la metacognición; por ello gestionar adecuadamente sus estrategias de aprendizaje es imperante para procurar la gestión de esas habilidades en el proceso de formación, considerando que la población estudiantil actual se encuentra dentro de la generación Millennials, cuya característica es la hiper-conectividad en la web, por lo que al no encontrarse dentro de este medio el estudiante no responderá adecuadamente a las posturas tradicionalistas en su aprendizaje, por esta razón surge la oportunidad y necesidad de abordar el proceso educativo desde otras perspectivas. La presente propuesta nace en la necesidad de implementar los recursos de la web combinados con los criterios gamificados con el fin de innovar el aprendizaje teórico y práctico de la asignatura de psicología social, dadas las características teóricas básicas en la formación de un psicólogo al cursar el segundo semestre en la carrera de psicología.
Con el presente estudio se buscó estimar si el uso de las estrategias de aprendizaje propuestas en el cuestionario de la Escala de Evaluación de Estrategias de Aprendizaje para estudiantes universitarios (CEVEAPEU), elaborado por Gargallo et al., (2009), pueden incentivar el aprendizaje de la asignatura a través de los cuestionamientos ejecutados en el interfaz gamificada.

La WEB fue desarrollada bajo software PHP y MYSQL, esta aplicación contenía actividades que potencian el uso de estrategias cognitivas y afectivas en el aprendizaje de la asignatura basado en un comportamiento motivacional, bidireccional y de mejora significativa de proceso enseñanza aprendizaje cuyo fundamento esencial es la gamificacion de las actividades de aprendizaje. La hipótesis establecida para esta investigación fue: $\mathrm{La}$ implementación de una interfaz gamificada en la asignatura de psicología social influye significativamente en el uso de las estrategias de aprendizaje en los estudiantes de la Universidad Tecnológica Indoamérica (UTI).

\section{Estrategias de aprendizaje}

Aprender es inherente al ser humano al tener un diseño cerebral predispuesto a ello desde que nace, a lo largo de la historia este desarrollo se ha ido perfeccionando y nuestro abanico de conocimientos ha logrado cambiar el estilo de vida, al punto de depender de herramientas tecnológicas para acciones rutinarias. Gargallo, (2009) menciona que cada individuo tiene una forma propia de aprender y los aportes teóricos indican un particular estilo de aprendizaje, donde las estrategias desplegadas permitirán en mayor o menor grado su gestión a la hora de asimilar un conocimiento, el uso de la tecnología vincula a su consumo una particularidad de dependencia que nos permite participar de espacios virtuales o crear espacios propios, exhibir un lenguaje propio y hasta esbozar espacios personales con inventiva propia, la cual rompe las barreras de la imaginación como nunca antes en la historia.

La forma de asimilar un aprendizaje está adaptado a cualquier ciencia del conocimiento, desde las ciencias abstractas, hasta las ciencias experimentales. Ser conscientes del tipo de habilidades 
que desplegamos para ejecutar una estrategia de aprendizaje es vital para administrar de forma adecuada el tiempo, la relevancia del conocimiento y su practicidad esto facilita la instrucción de forma lógica y secuencial volviendo el estudio una actividad concreta y centrada en los aspectos más relevantes y útiles a los intereses de cada individuo.

Para Esteban \& Zapata (2016), las destrezas de aprendizaje representan un procedimiento de operaciones ante un trabajo que requiere una actividad cognitiva que implica aprendizaje; es decir, se activa el proceso de ejecutar tareas a través de técnicas que ayudan a la síntesis de información, la resolución de problemas, al análisis de datos, la relación de conceptos y la retención de información. Toda estrategia implica una actividad cognitiva consciente mediada por aspectos cognitivos, afectivos y motivacionales los cuales deben administrarse adecuadamente, tarea no siempre fácil ya que no todos conocen de este recurso ni saben cómo utilizarlos de forma apropiada y oportuna.

Para Sánchez (2014), las estrategias presentan atributos esenciales de carácter intencional y propositivo procesado por el educando al realizar ciertos patrones conductuales que accionan la consecución de un fin específico. Donde la motivación por aprender algo es el motor que activa al cerebro, ya que provee el combustible a la curiosidad y la chispa que despliega nuestro empeño en alcanzar algo.

Para Gargallo (2009) involucra la disposición organizada, juiciosa y deliberada de acciones ejecutadas por el aprendiz con el objetivo de aprender. La presente investigación asume esta postura, ya que el dicente que asume una organización consciente en el procesamiento de la información, gestiona su organización, está motivado a aprender. Asumir un rol protagónico confluye en un estudio eficiente y eficaz. La llave del proceso es la motivación del sujeto en el deseo que tiene de formarse; es aquí donde interviene la administración pertinente, reflexiva y lógica en la implementación de sus tácticas de instrucción.

Estas pericias de aprendizaje son clasificadas por Gargallo (2009) como: afectivas, de apoyo y control, estas incluyen la voluntad del aprendiz, su autonomía por aprender y suscitan su capacidad para reflexionar; en la que también se observa las estrategias motivacionales, que dan especial énfasis al valor de la tarea, la atribución a la misma, la autoeficacia, las expectativas y la concepción de la inteligencia; dentro de los componentes afectivos se observa el estado físico-anímico del dicente, el manejo de la ansiedad; las destrezas metacognitivas adscriben el conocimiento, el control (que incluye la organización, valoración, inspección y ordenación) y las habilidades de control del contexto (socialización y administración de recursos).

Las estrategias cognitivas se adscriben al proceso de aprendizaje, representan tácticas en el conocimiento y la búsqueda de información que se busca alcanzar; contiene además estrategias en el procesamiento y uso de la indagación como: adquirir, codificar, elaborar y organizar la información, la repetición, el almacenamiento, la evocación. El autor expone que al explorar estas cualidades en las estrategias de aprendizaje valoramos su uso, pero a la vez se puede orientar su uso. Es pertinente resaltar que la población objeto de estudio donde se aplicó la estandarización del instrumento está conformada por estudiantes universitarios. La acepción teórica se basa en el estudiante, el cual opera bajo su voluntad, dinamiza su capacidad y autonomía, suscita el auto educarse, convirtiéndose en el responsable de lo que aprende para alcanzar metas propuestas. (Sánchez, 2015)

\section{Tecnología Educativa}

Por tecnología educativa se entenderá la adaptación y utilización de las TIC en los diferentes momentos del proceso educativo. Orientada a mediar el proceso educativo, de conformidad al contexto sociocultural e histórico, que gestione su implementación en un diseño propio, la evaluación y una retroalimentación adaptada al proceso de ejecución como a los entornos virtuales de aprendizaje (Cabero, 2017). Los procesos mediados por el uso de las TIC generan impacto dada su dinámica y facilidades en la retroalimentación en tiempo real. Aquí la tecnología educativa puede adaptarse a los diferentes momentos del proceso educativo, adecuado al contexto en el cual se utilizará, con un diseño, ejecución y evaluación asociado tanto al entorno material o virtual para 
el aprendizaje (Arteaga \& Basurto, 2017).

Por lo expuesto el docente debe aprovechar las bondades de estos sistemas con el fin de activar la motivación extrínseca como un medio que permita al estudiante entornos afines a su cotidianidad tecnológica. El proceso de retroalimentación en tiempo real también permite que el estudiante gestione correctivos a lo que aprende y reestructure la información, el docente guía su aprendizaje hacia el desarrollo de habilidades que le permitan resolver un problema específico del entorno.

\section{Gamificación}

Las aplicaciones gamificadas en el uso de las tecnologías han originado cambios que han transformado la forma comunicativa en nuevos modos de gestión, fundamentalmente en el accionar escolar, ya sea a nivel individual como grupal esto obedece a que las mismas incorporan en su estructura la dinámica del juego, la motivación y los criterios del condicionamiento operante. Sus aplicaciones inventan nuevos modos de comunicación interactiva con instrumentos tecnológicos que superan las barreras espacio-temporales en su acceso y en la forma de presentar la información (Sánchez, 2015).

Las estrategias de gamificación son gestores en el comportamiento de las personas al influir en este, independientemente de otros objetivos secundarios, el usuario puede conseguir un disfrute durante la realización de la actividad del juego (Díaz \& Troyano, 2013). La conducta del usuario adquiere una dinámica, donde la estimulación por conseguir la meta activa procesos neurológicos que potencian el poder alcanzar algo, un combustible que incentiva la iniciativa de logro. Al utilizar procesos gamificados en el aprendizaje se busca que los estudiantes estudien bajo el enfoque del entretenimiento pero guiados hacia un fin específico; el cual le ofrece premios, prestigio entre sus compańeros, con ventajas tanto para estudiantes como para maestros, ya que se asocian en un apoyo mutuo que facilita el estudio de la asignatura, con objetivos claros en base a retos que resuelven un problema que aporta al perfil de egreso de su carrera, pero esto a la vez demanda el cumplimiento de reglas y al final del transcurso se obtienen una ganancia por los esfuerzos realizados ya sean tangibles como intangibles (Landers \& Callan, 2011). El proceso gestiona la autoeducación, la necesidad de resolver actividades prácticas que favorecen la toma de decisiones. Enseñar con juegos refuerza el aprendizaje, ya que cuando se aprende, la diversión aumenta la motivación y reduce el estrés. Es sustancial destacar como desventaja la presencia de grupos heterogéneos, ya que no todos se involucran en igual forma en el proceso de estudio con esmero y empeño (Gallego et al., 2014). Sin embargo, aquí está el desafío del docente en buscar que el grupo procure alcanzar el resultado de aprendizaje contemplado en el currículo, el tipo de recompensas que utilice permitirán a sus participantes acumular puntos por los aprendizajes adquiridos, a los cuales se les puede asignar un valor cuantitativo a cada una de las operaciones del proceso; le permite al estudiante escalar niveles para llegar a la siguiente fase; las bonificaciones aumentan a medida que se siguen cumpliendo objetivos de aprendizaje planteados a lo largo del juego y al final se pueden trabajar en retos individuales o grupales que finalmente nos llevan a la meta. Así, la gamificación como destreza de instrucción, transpone el funcionamiento de los juegos al perímetro educativo para a corto plazo mejorar los resultados de aprendizaje de la asignatura y a largo plazo el desarrollo de habilidades que le permitan resolver problemas del contexto, modelar un comportamiento ético en la resolución de los mismos (Gaitán, 2013). Aprovechar este tipo de recursos nos conecta como docentes a nuestros estudiantes, el aprendizaje se enrique en otro contexto y la motivación acciona el interés por aprender.

\section{Rol de docente en el aprendizaje de un millenials}

El uso de la tecnología obliga a repensar nuestro rol en el espacio educativo, la generación a la que educamos presenta características sociales, comunicativas diferentes a las de sus docentes. Representa un desafío generacional para docentes y estudiantes, sin embargo, hay condiciones básicas que no se pueden dejar de observar en la implementación de este proceso como el dominio básico de los criterios referentes a la asignatura, el 
uso de metodologías didácticas en la clase, el manejo de las TIC y la humildad de aceptar aquello que no se conoce.

La educación del siglo XXI demanda la gestión pertinente del conocimiento en miras a procurar la competitividad en un contexto social el cual demanda eficacia y eficiencia. Una educación equitativa con iguales oportunidades de acceso brinda a sus miembros la oportunidad de incorporarse al mercado laboral en igualdad de condiciones, (Aguerrondo, 2017). El proceso de aprendizaje mediado por las tecnologías demanda de sus usuarios un conocimiento y la administración de sus espacios para aprovechar al máximo sus ventajas. Para Requena, (2016) los roles de los gestores en el aprendizaje se gestionan ahora en matices de aprendizaje compartido, donde los conocimientos de unos y otros convergen con un propósito practico, coherente y anexo a resolver los problemas de un entorno social que presenta particularidad en el acceso al conocimiento, la participación, y la gestión de su uso; pero además esto confluyen en una óptica social que se mira diferente no solo por las instancias culturales, la condición económica, la educación sino también el valor de la consciencia ciudadana de sus miembros, Rodríguez (2017). De lo expuesto compete a cada maestro la tarea de concretar un aprendizaje práctico a la futura labor productiva, donde sin duda el avance tecnológico medie los procesos de su propia gestión, los estudiantes de hoy son nativos tecnológicos y lo pertinente de nuestro rol es utilizar esos medios para gestionar un aprendizaje efectivo.

\section{Desarrollo de la interfaz gamificada}

Algunas de las TIC empleadas en el desarrollo de la interfaz gamificada son PHP y MYSQL. El PHP es un lenguaje de scripting en servidor y una poderosa herramienta para hacer páginas web dinámicas e interactivas. PHP es una alternativa ampliamente utilizada, gratuita y eficiente para los competidores, como ASP de Microsoft. Los archivos PHP pueden contener texto, HTML, CSS, JavaScript y código PHP. El código PHP se ejecuta en el servidor y el resultado se devuelve al navegador como HTML simple. Los archivos PHP tienen la extensión "php” (Cobo et al.,
2005). Este tipo de páginas resultantes al contener solo código HTML es compatible con otro tipo de navegadores.

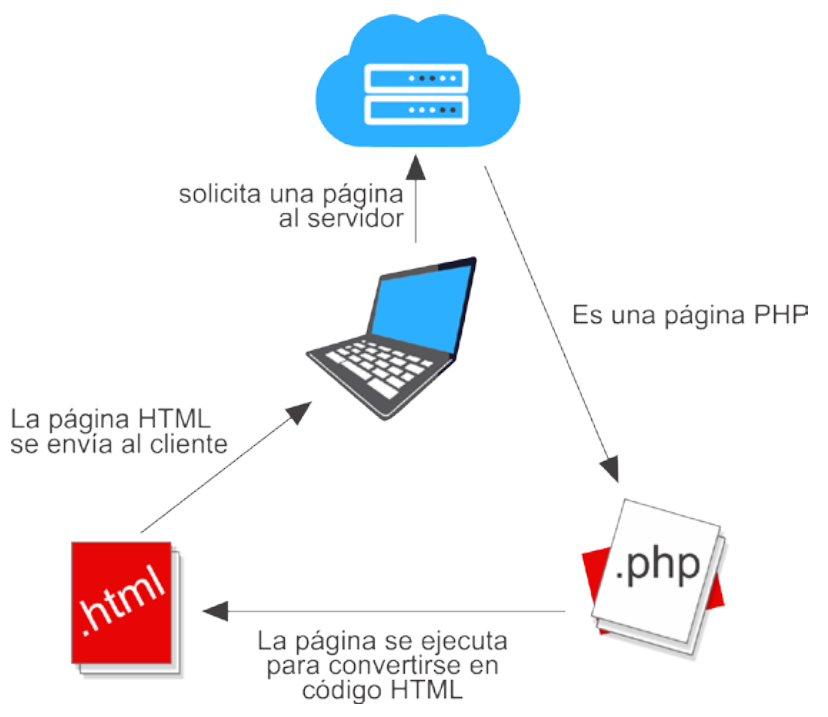

Figura 1. Compatibilidad de PHP Fuente: Alvarez, 2011

La aplicación del interfaz con la metodología de gamificación potencia el aprendizaje de una manera amigable y bidireccional, porque ayuda al usuario en la motivación, la concentración, el compromiso, el esfuerzo y la fidelización, entre otros valores. Es aplicable a diversos ámbitos académicos, la base de datos MYSQL es adaptable a cualquier materia a dictarse lo que contribuye a mejorar y retroalimentar oportunamente el proceso enseńanza - aprendizaje, así como el rendimiento escolar.

MySQL representa un sistema cuya función es la de gestionar base de datos, es uno de los más usados y reconocidos para el desarrollo web, representa una mezcla de $\mathrm{C}_{\text {y }} \mathrm{C}++_{+}$, es uno de los cuatro componentes del paquete LAMP, facilita la conjunción con otro tipo de servidores web, se relaciona con facilidad con aplicaciones web o CMS para sitios online, como wordpress, drupla, joomla, entre otros, muy ligado a PHP. Se destaca por su rapidez de lectura sobre todo para motores como MylSAM; una desventaja es que sea cual sea el entorno y su objetivo es necesario monitorizar el rendimiento para poder corregir los errores, tanto de programación como de SQL. (Welling \& Thomson, 2003) 
Como los métodos tradicionales no se adaptaban a las exigencias del proyecto se utilizó la metodología de desarrollo RUP (Proceso Unificado Racional) que reúne elementos genéricos de otros modelos que ayudan en el desarrollo de software robusto y costeable en tiempos cortos mejorando la seguridad. Se desarrolló por fases: a) Inicio. - tiene como fin definir los elementos internos y externos para la identificación de riesgos asociados. b) elaboración. - Se diseña la solución preliminar al problema planteado y se seleccionan los casos de uso. c) construcción. - Codifica y se prueba el software, para la integración de elementos al sistema. d) transición. - realizar un pilotaje que permita la identificación de errores y defectos encontrados durante las pruebas de aceptación la retroalimentación y soporte técnico necesario.

La aplicación WEB fue desarrollada pensando en ser adaptable y reutilizable, utiliza software libre (PHP y MYSQL) que economiza el desarrollo de la misma, siendo una herramienta complementaria al aula virtual. A este proceso se implanta la metodología de gamificación para que el estudiante previo a la revisión de un subtema de estudio, responda a un cuestionario de preguntas, realización de juegos interactivos; ejecutadas a través de la aplicación web gamificada, cada actividad ejecutada proporciona una retroalimentación diferida, la cual al final proporciona premios de incentivo. Junto a las actividades desarrolladas en el interfaz, se busca también su implementación en la clase en la fase de construcción del conocimiento al ejecutar los talleres prácticos en la resolución de casos.

\section{Materiales y Métodos}

\section{Participantes}

El presente estudio ejecuto un proceso de entrenamiento en estrategias de aprendizaje, a través del desarrollo de actividades ejecutadas en un interfaz gamificada durante el tratamiento de los temas de clase en el abordaje de la asignatura de psicología social durante el periodo académico septiembre-marzo del 2018 en la carrera de psicología, se contó con dos grupos uno control y otro experimental a quienes se les instruyo en el uso de estrategias de aprendizaje a través de actividades ejecutadas en un interfaz gamificada; el cual fue desarrollado con la finalidad de ser un medio que potencialice el uso de estrategias de aprendizaje. La población de estudio representaron los estudiantes de segundo nivel de la carrera de Psicología de la UTI, que asisten a la asignatura de psicología social, al ser una población pequeña inferior a 100 la muestra se estima al total de la población, en ellos se aplicó el muestreo probalístico de grupo - control, en el cual se estableció el grupo experimental conformado por 21 estudiantes de los cuales 16 son mujeres y 5 hombre, a quienes se le aplicó la gamificación como estrategia de aprendizaje; y el de control que mantuvo la enseńanza tradicional, que fueron 26 estudiantes 14 mujeres y 12 hombres. Dentro de los criterios de ética aplicados para esta investigación se usaron el consentimiento informado, la edad de los estudiantes fue de 18 a 24 años, nivel socioeconómico medio y provenientes del sector urbano.

\section{Instrumento}

Previo al acceso y uso del interfaz gamificada se determinó aplicar a los estudiantes la Escala de Evaluación de Estrategias de Aprendizaje para estudiantes universitarios (CEVEAPEU), elaborado por Gargallo et al., (2009), quien destaca que las misma son herramientas para aprender, las cuales tienen influencia en el logro académico. En este sentido, el autor determina que el uso de estas habilidades en el aprendizaje puede predecir un éxito académico. En tal virtud el presente instrumento satisface la necesidad de proporcionar una medición confiable y válida para la variable en estudio y para el contexto hispano universitario.

El CEVEAPEU cuenta entre sus antecedentes con el Learning and Study Strategies Inventory (LASSI), Weinstein et al., (1987), que contiene una escala de concentración, pero omite algunas estrategias cognitivas y metacognitivas importantes incluidas en el CEVEAPEU. También se deriva del Motivated Strategies for Learning Questionnaire (MSLQ) (Pintrich et al., 1991), donde Gargallo et al. (2009) creen que las subescalas motivacionales no evalúan algunas estrategias importantes como atribuciones internas y externas, estado físi- 
co y anímico, y estrategias cognitivas relacionadas con la memorización y la transferencia. El CEVEAPEU consta de 88 declaraciones contenidas en dos escalas y seis subescalas, valoradas mediante una escala Likert que va desde muy de acuerdo hasta muy en desacuerdo. La primera escala aprecia estrategias afectivas, de apoyo y control, la cual consta de cincuenta y tres ítems conformado por cuatro subescalas de estrategias: motivacionales, componentes afectivos, metacognitivas y de control del contexto, la interacción social y el manejo de recursos. La segunda escala contempla pericias relacionadas para el procesamiento de la información con treinta y cinco ítems e incluye dos subescalas: de búsqueda y selección de información, y estrategias de procesamiento y uso de la información, la validación de la escala reporta una validez de constructo, contrastada por la valoración de los jueces y por los análisis factoriales, un alto nivel de consistencia interna corroborado por el análisis factorial a cada subescala, la validez predictiva se contrasta con correlaciones positivas y regresión múltiple aceptables Gargallo et al., (2009).

\begin{tabular}{|c|c|c|}
\hline \multirow{4}{*}{$\begin{array}{l}\text { 1. Estrategias } \\
\text { afectivas, de apoyo y } \\
\text { control }\end{array}$} & 1.1. Estrategias motivacionales & $\begin{array}{l}\text { 1.1.1. Motivación } \\
\text { 1.1.2. Valor de la tarea } \\
\text { 1.1.3. Persistencia en la tarea } \\
\text { 1.1.4. Atribuciones } \\
\text { 1.1.5. Autoeficacia y expectativas } \\
\text { 1.1.6. Concepción de la inteligencia como modificable }\end{array}$ \\
\hline & 1.2. Componentes afectivos & $\begin{array}{l}\text { 1.2.1. Estado fisico y anímico } \\
\text { 1.2.2. Ansiedad }\end{array}$ \\
\hline & 1.3. Estrategias metacognitivas & $\begin{array}{l}\text { 1.3.1. Conocimiento } \\
\text { 1.3.2. Control (Estrategias de planificación, evaluación, } \\
\text { control y regulaciön) }\end{array}$ \\
\hline & $\begin{array}{l}\text { 1.4. Estrategias de control del contexto, } \\
\text { interacción social y manejo de recursos }\end{array}$ & $\begin{array}{l}\text { 1.4.1. Control del contexto } \\
\text { 1.4.2. Habilidades de interacción social y aprendizaje con } \\
\text { compañeros }\end{array}$ \\
\hline \multirow[b]{2}{*}{$\begin{array}{l}\text { 2. Estrategias } \\
\text { cognitivas } \\
\text { (relacionadas con el } \\
\text { procesamiento de la } \\
\text { información }\end{array}$} & $\begin{array}{l}\text { 2.1. Estrategias de búsqueda, recogida y } \\
\text { selección de información y uso de la información }\end{array}$ & $\begin{array}{l}\text { 2.1.1. Conocimiento de fuentes } \\
\text { 2.1.2. Selección de información }\end{array}$ \\
\hline & $\begin{array}{l}2.2 \text { Estrategias de procesamiento y uso de la } \\
\text { información }\end{array}$ & $\begin{array}{l}\text { 2.2.1. Adquisición de información } \\
\text { 2.2.2. Codificación, elaboración y organización de la y uso } \\
\text { de la información } \\
\text { 2.2.3. Personalización y creatividad } \\
\text { 2.2.4. Repetición y almacenamiento } \\
\text { 2.2.5. Recuperación de la información } \\
\text { 2.2.6. Uso y transferencia de la información adquirida }\end{array}$ \\
\hline
\end{tabular}

Figura 2. Clasificación de las estrategias según Gargallo. Fuente: Gargallo, Almerich, Suárez, \& García, 2012

\section{Tipo y diseño de estudio}

La metodología se basó en un estudio longitudinal con un diseño cuasiexperimental, con grupo de control, al grupo experimental se le realizó un pre y pos test respecto al uso de estrategias de aprendizaje, el aspecto mediado a la gestión de estas habilidades consistió en uso de una plataforma gamificada que mediante el análisis de estadística inferencial demuestra que el uso del interfaz incide en las estrategias de aprendizaje.

\section{Procedimiento}

El estudio consideró un proceso de tres etapas: en la primera etapa se procedió a la creación de un panel de acceso en PHP utilizando como gestor de base de datos MYSQL, en el cual los usuarios "Docentes o alumnos" ingresan su identificación y contraseña para su registro. El sistema verifica si el usuario es un docente o un alumno mediante el registro de rol de la pantalla de usuario correspondiente a los alumnos, es un menú, en forma de tablero de monopolio que contiene una serie de juegos, cada juego contiene preguntas a ser resueltas por el usuario, pero a su vez ofrece una retroalimentación diferida inmediata, la cual es activada después de una respuesta correcta, lo cual permite tener acceso a otro juego de mayor jerarquía en el contenido de la información. El rol de administrador es dado para los docentes quienes gestionan los niveles y cuestionarios de la plataforma, los mismos que pueden partir desde conocimientos generales hasta la resolución de casos. El sistema genera reportes por estudiante sobre la utilización de juegos, la posición jerárquica que ocupa el jugador frente a otros y las respuestas del aprendizaje para cada uno de los temas abordados. La intención de cada juego obliga al estudiante a demostrar el desarrollo de más de dos estrategias de aprendizaje en el proceso de su estudio. En la misma también se realizó la selección de tres temas de la clase a) el comportamiento social, b) las actitudes y c) los prejuicios en el tratamiento de la asignatura de psicología social, los cuales fueron considerados por la programación de la asignatura durante aproximadamente un mes de estudio. Se conformó dos grupos que corresponde a dos paralelos conformados por la misma proporción de estudiantes. El primero (experimental) está conformado por 24 estudiantes entre hombres y mujeres, a quienes se le aplicó previo al uso del interfaz gamificada la escala de estrategias de aprendizaje CEVEAPEU, mientras que al segundo grupo (control) tan solo se evaluó las estrategias de aprendizaje con la misma escala y la metodología de aprendizaje no hubo cambio en ningún aspecto. La segunda etapa contempló 
realizar el mismo proceso de tareas, elaboración de talleres, exposiciones y evaluaciones para los dos grupos. Para la tercera etapa se considera como única variación el uso del uso del interfaz gamificada, para el grupo experimental, el cual consistía en realizar y resolver una serie de juegos; al finalizar esta etapa se considera los reportes generales del grupo experimental. El tiempo de uso en la plataforma depende de cada usuario. Al final del proceso todos los estudiantes lograron resolver los juegos presentes en el interfaz.

\section{Resultados}

En la Tabla 1 y 2 se muestra los resultados del desempeño de los estudiantes de grupo experimental y control, en relación con los temas de comportamiento social, actitudes y prejuicios, validando que mejora el desempeño académico en el grupo experimental en relación del pre y post test.

\section{Tabla 1}

Utilización de la interfaz gamificada y desempeño acadé-

GAMIFICACION*\$PRETEST*GRUPO Tabulación cruzada

\begin{tabular}{|c|c|c|c|c|c|c|c|c|}
\hline \multirow{2}{*}{$\begin{array}{l}\text { Grupo de } \\
\text { estudio }\end{array}$} & \multirow{2}{*}{\multicolumn{2}{|c|}{$\begin{array}{c}\text { Uso de } \\
\text { Gamificación }\end{array}$}} & \multicolumn{5}{|c|}{ TEMAS PRETEST } & \multirow{2}{*}{ Total } \\
\hline & & & 1.00 & 2.00 & 3.00 & 4.00 & 5.00 & \\
\hline \multirow{2}{*}{ Experimental } & \multirow{2}{*}{ SI } & $\mathrm{N}^{\circ}$ & - & 3 & 9 & 5 & 4 & 21 \\
\hline & & $\%$ & - & 14.3 & 42.9 & 23.8 & 19.0 & 100 \\
\hline \multirow{2}{*}{ Control } & \multirow{2}{*}{ NO } & N. ${ }^{\circ}$ & 7 & 6 & 10 & 1 & 2 & 26 \\
\hline & & $\%$ & 26.9 & 23.1 & 38.5 & 3.8 & 7.7 & 100 \\
\hline
\end{tabular}

\section{mico}

${ }^{*}$ Los porcentajes y los totales se basan en los encuestados Fuente: Evaluación general de los 2 grupos de estudio. Elaborado por: Ramírez \& Mayorga \& Lara, 2019

Tabla 2

\begin{tabular}{|c|c|c|c|c|c|c|c|}
\hline \multicolumn{8}{|c|}{ GAMIFICACION*\$POSTTEST*GRUPO Tabulación cruzada } \\
\hline \multirow{2}{*}{$\begin{array}{l}\text { Grupo de } \\
\text { estudio }\end{array}$} & \multirow{2}{*}{\multicolumn{2}{|c|}{$\begin{array}{c}\text { Uso de } \\
\text { Gamificación }\end{array}$}} & \multicolumn{4}{|c|}{ TEMAS POSTTEST } & \multirow{2}{*}{ Total } \\
\hline & & & 6.00 & 7.00 & 800 & 9.00 & \\
\hline \multirow{2}{*}{ Experimental } & \multirow{2}{*}{ SI } & $\mathrm{N} .^{\circ}$ & 1 & 16 & 18 & 28 & 21 \\
\hline & & $\%$ & 4.8 & 76.2 & 85.7 & 133.3 & 100 \\
\hline \multirow{2}{*}{ Control } & \multirow{2}{*}{ NO } & $\mathrm{N} .^{\circ}$ & 6 & 20 & 5 & - & 26 \\
\hline & & $\%$ & 23.1 & 76.9 & 19.2 & - & 100 \\
\hline
\end{tabular}

Utilización de la interfaz Gamificada y rendimiento

${ }^{*}$ Los porcentajes y los totales se basan en los encuestados Fuente: Evaluación general de los 2 grupos de estudio. Elaborado por: Ramírez \& Mayorga \& Lara, 2019

Como se puede demostrar el desempeño académico mejora significativamente en el grupo experimental son más altos que el grupo experimental alcanzando notas de 9 puntos, versus los del grupo control que alcanza notas de 8 puntos.

Tabla 3

Estrategia afectiva $(\mathrm{MDA}=$ Muy de acuerdo, $\mathrm{DA}=\mathrm{De}$ acuerdo, $\mathrm{I}=$ Indeciso, $\mathrm{ED}=$ En desacuerdo, $\mathrm{MED}=$ Muy en desacuerdo.

\begin{tabular}{|c|c|c|c|c|c|c|c|c|}
\hline \multicolumn{9}{|c|}{ J1*\$AFECTIVAS*GRUPO tabulación cruzada* } \\
\hline \multirow{2}{*}{\multicolumn{3}{|c|}{ Grupo de estudio }} & \multicolumn{5}{|c|}{ ESTRATEGIAS AFECTIVAS } & \multirow[t]{2}{*}{ Total } \\
\hline & & & MDA & $\mathrm{DA}$ & I & ED & MED & \\
\hline \multicolumn{9}{|c|}{ JUEGOS DE MEMORIA } \\
\hline \multirow{6}{*}{$\begin{array}{l}\text { Experi- } \\
\text { mental }\end{array}$} & \multirow{2}{*}{$\begin{array}{l}\text { Total- } \\
\text { mente }\end{array}$} & $\mathrm{N} .^{\circ}$ & 15 & 16 & 11 & 4 & 2 & 12 \\
\hline & & $\%$ & 71.4 & 76.2 & 52.4 & 19.0 & 9.5 & 57.1 \\
\hline & \multirow{2}{*}{$\begin{array}{l}\text { Parcial- } \\
\text { mente }\end{array}$} & $\mathrm{N} .^{\circ}$ & 10 & 13 & 7 & 4 & 2 & 9 \\
\hline & & $\%$ & 47.4 & 61.9 & 33.3 & 19.0 & 9.5 & 42.9 \\
\hline & \multirow{2}{*}{ TOTAL } & N. ${ }^{\circ}$ & 25 & 29 & 18 & 8 & 4 & 21 \\
\hline & & $\%$ & 119.0 & 138.1 & 85.7 & 38.1 & 19.0 & 100.0 \\
\hline \multirow{2}{*}{ Control } & \multirow{2}{*}{ Nada } & $\mathrm{N} .^{\circ}$ & 12 & 18 & 29 & 25 & 20 & 26 \\
\hline & & $\%$ & 46.2 & 69.2 & 111.5 & 96.2 & 76.9 & 100.0 \\
\hline
\end{tabular}

\section{JUEGOS MOTIVACIONALES}

\begin{tabular}{|c|c|c|c|c|c|c|c|c|}
\hline \multirow{6}{*}{$\begin{array}{l}\text { Experi- } \\
\text { mental }\end{array}$} & \multirow{2}{*}{$\begin{array}{l}\text { Total- } \\
\text { mente }\end{array}$} & $\mathrm{N} .^{\circ}$ & 8 & 13 & 8 & 5 & 2 & 9 \\
\hline & & $\%$ & 38.1 & 61.9 & 38.1 & 23.8 & 9.5 & 42.9 \\
\hline & \multirow{2}{*}{$\begin{array}{l}\text { Parcial- } \\
\text { mente }\end{array}$} & $\mathrm{N} .^{\circ}$ & 17 & 16 & 10 & 3 & 2 & 12 \\
\hline & & $\%$ & 81.0 & 76.2 & 47.6 & 14.3 & 9.5 & 57.1 \\
\hline & \multirow{2}{*}{ TOTAL } & $\mathrm{N} .^{\circ}$ & 25 & 29 & 18 & 8 & 4 & 21 \\
\hline & & $\%$ & 119.0 & 138.1 & 85.7 & 38.1 & 19.0 & 100.0 \\
\hline \multirow{2}{*}{ Control } & \multirow{2}{*}{ Nada } & $\mathrm{N} .^{\circ}$ & 12 & 18 & 29 & 25 & 20 & 26 \\
\hline & & $\%$ & 46.2 & 69.2 & 111.5 & 96.2 & 76.9 & 100.0 \\
\hline \multicolumn{9}{|c|}{ JUEGOS DE CONCENTRACIÓN } \\
\hline \multirow{6}{*}{$\begin{array}{l}\text { Experi- } \\
\text { mental }\end{array}$} & \multirow{2}{*}{$\begin{array}{l}\text { Total- } \\
\text { mente }\end{array}$} & $\mathrm{N} .^{\circ}$ & 2 & 7 & 4 & 5 & 0 & 9 \\
\hline & & $\%$ & 9.5 & 33.3 & 19.0 & 23.8 & 0.0 & 42.9 \\
\hline & \multirow{2}{*}{$\begin{array}{l}\text { Parcial- } \\
\text { mente }\end{array}$} & $\mathrm{N} .^{\circ}$ & 3 & 8 & 4 & 6 & 3 & 12 \\
\hline & & $\%$ & 14.3 & 38.1 & 19.0 & 28.6 & 14.3 & 57.1 \\
\hline & \multirow{2}{*}{ TOTAL } & $\mathrm{N} .^{\circ}$ & 5 & 15 & 8 & 11 & 3 & 21 \\
\hline & & $\%$ & 23.8 & 71.4 & 38.1 & 52.4 & 14.3 & 100.0 \\
\hline \multirow{2}{*}{ Control } & \multirow{2}{*}{ Nada } & $\mathrm{N} .^{\circ}$ & 8 & 11 & 15 & 9 & 9 & 26 \\
\hline & & $\%$ & 30.8 & 42.3 & 57.7 & 34.6 & 34.6 & 100.0 \\
\hline \multicolumn{9}{|c|}{ JUEGOS DE ESTRATEGIA } \\
\hline \multirow{6}{*}{$\begin{array}{l}\text { Experi- } \\
\text { mental }\end{array}$} & \multirow{2}{*}{$\begin{array}{l}\text { Total- } \\
\text { mente }\end{array}$} & $\mathrm{N}^{\circ}$ & 11 & 17 & 10 & 5 & 1 & 11 \\
\hline & & $\%$ & 52.4 & 81.0 & 47.6 & 23.8 & 4.8 & 52.4 \\
\hline & \multirow{2}{*}{$\begin{array}{l}\text { Parcial- } \\
\text { mente }\end{array}$} & $\mathrm{N} .^{\circ}$ & 14 & 12 & 8 & 3 & 3 & 10 \\
\hline & & $\%$ & 66.7 & 57.1 & 38.1 & 14.3 & 14.3 & 47.6 \\
\hline & \multirow{2}{*}{ TOTAL } & $\mathrm{N} .^{\circ}$ & 25 & 29 & 18 & 8 & 4 & 21 \\
\hline & & $\%$ & 119.0 & 138.1 & 85.7 & 38.1 & 19.0 & 100.0 \\
\hline \multirow{2}{*}{ Control } & \multirow{2}{*}{ Nada } & $\mathrm{N} .^{\circ}$ & 12 & 18 & 29 & 25 & 20 & 26 \\
\hline & & $\%$ & 46.2 & 69.2 & 111.5 & 96.2 & 76.9 & 100.0 \\
\hline
\end{tabular}

ISSN 2313-7878. Hamut'ay 6(3). Setiembre-diciembre 2019. Págs. 62-80

70 


\begin{tabular}{|c|c|c|c|c|c|c|c|c|}
\hline \multicolumn{9}{|c|}{ J1*\$AFECTIVAS*GRUPO tabulación cruzada* } \\
\hline \multirow{2}{*}{\multicolumn{3}{|c|}{ Grupo de estudio }} & \multicolumn{5}{|c|}{ ESTRATEGIAS AFECTIVAS } & \multirow[t]{2}{*}{ Total } \\
\hline & & & MDA & DA & I & ED & MED & \\
\hline \multicolumn{9}{|c|}{ JUEGOS DE LOGRO } \\
\hline \multirow{6}{*}{$\begin{array}{l}\text { Experi- } \\
\text { mental }\end{array}$} & \multirow{2}{*}{$\begin{array}{l}\text { Total- } \\
\text { mente }\end{array}$} & $\mathrm{N} .^{\circ}$ & 14 & 15 & 11 & 5 & 3 & 12 \\
\hline & & $\%$ & 66.7 & 71.4 & 52.4 & 23.8 & 14.3 & 57.1 \\
\hline & \multirow{2}{*}{$\begin{array}{l}\text { Parcial- } \\
\text { mente }\end{array}$} & $\mathrm{N} .^{\circ}$ & 11 & 14 & 7 & 3 & 1 & 9 \\
\hline & & $\%$ & 52.4 & 66.7 & 33.3 & 14.3 & 4.8 & 42.9 \\
\hline & \multirow{2}{*}{ TOTAL } & $\mathrm{N} .^{\circ}$ & 25 & 29 & 18 & 8 & 4 & 21 \\
\hline & & $\%$ & 119.0 & 138.1 & 85.7 & 38.1 & 19.0 & 100.0 \\
\hline \multirow{2}{*}{ Control } & \multirow{2}{*}{ Nada } & $\mathrm{N} .^{\circ}$ & 12 & 18 & 29 & 25 & 20 & 26 \\
\hline & & $\%$ & 46.2 & 69.2 & 111.5 & 96.2 & 76.9 & 100.0 \\
\hline
\end{tabular}

${ }^{*}$ Los porcentajes y los totales se basan en los encuestados Fuente: Evaluación general de los 2 grupos de estudio. Elaborado por: Ramírez \& Mayorga \& Lara, 2019

De otro lado cabe resaltar que la Tabla 4 indica los juegos que más activan la estrategia cognitiva donde los juegos de memoria, motivacionales y de concentración inciden en la misma.

\section{Tabla 4}

Estrategia cognitiva $\quad(\mathrm{MDA}=$ Muy de acuerdo, $\mathrm{DA}=\mathrm{De}$ acuerdo, $\mathrm{I}=$ Indeciso, $\mathrm{ED}=$ En desacuerdo, $\mathrm{MED}=$ Muy en desacuerdo.

\begin{tabular}{|c|c|c|c|c|c|c|c|c|}
\hline \multicolumn{9}{|c|}{ J1*\$COGNITIVAS*GRUPO tabulación cruzada* } \\
\hline \multirow{2}{*}{\multicolumn{3}{|c|}{ Grupo de estudio }} & \multicolumn{5}{|c|}{ ESTRATEGIAS COGNITIVAS } & \multirow[t]{2}{*}{ Total } \\
\hline & & & MDA & $\mathrm{DA}$ & I & ED & MED & \\
\hline \multicolumn{9}{|c|}{ JUEGOS DE MEMORIA } \\
\hline \multirow{6}{*}{$\begin{array}{l}\text { Experi- } \\
\text { mental }\end{array}$} & \multirow{2}{*}{$\begin{array}{l}\text { Total- } \\
\text { mente }\end{array}$} & N. ${ }^{\circ}$ & 3 & 8 & 5 & 7 & 1 & 12 \\
\hline & & $\%$ & 14.3 & 38.1 & 23.8 & 33.3 & 4.8 & 57.1 \\
\hline & \multirow{2}{*}{$\begin{array}{l}\text { Parcial- } \\
\text { mente }\end{array}$} & $\mathrm{N} .^{\circ}$ & 2 & 7 & 3 & 4 & 2 & 9 \\
\hline & & $\%$ & 9.5 & 33.3 & 14.3 & 19.0 & 9.5 & 42.9 \\
\hline & \multirow{2}{*}{ TOTAL } & $\mathrm{N} .^{\circ}$ & 5 & 15 & 8 & 11 & 3 & 21 \\
\hline & & $\%$ & $23.8 \%$ & 71.4 & 38.1 & 52.4 & 14.3 & 100.0 \\
\hline \multirow{2}{*}{ Control } & \multirow{2}{*}{ Nada } & $\mathrm{N} .^{\circ}$ & 8 & 11 & 15 & 9 & 9 & 26 \\
\hline & & $\%$ & 30.8 & 42.3 & 57.7 & 34.6 & 34.6 & 100.0 \\
\hline \multicolumn{9}{|c|}{ JUEGOS MOTIVACIONALES } \\
\hline \multirow{6}{*}{$\begin{array}{l}\text { Experi- } \\
\text { mental }\end{array}$} & \multirow{2}{*}{$\begin{array}{l}\text { Total- } \\
\text { mente }\end{array}$} & $\mathrm{N} .^{\circ}$ & 3 & 6 & 5 & 3 & 1 & 9 \\
\hline & & $\%$ & 14.3 & 28.6 & 23.8 & 14.3 & 4.8 & 42.9 \\
\hline & \multirow{2}{*}{$\begin{array}{l}\text { Parcial- } \\
\text { mente }\end{array}$} & $\mathrm{N}^{\circ}$ & 2 & 9 & 3 & 8 & 2 & 12 \\
\hline & & $\%$ & 9.5 & 42.9 & 14.3 & 38.1 & 9.5 & 57.1 \\
\hline & \multirow{2}{*}{ TOTAL } & $\mathrm{N} .^{\circ}$ & 5 & 15 & 8 & 11 & 3 & 21 \\
\hline & & $\%$ & $23.8 \%$ & 71.4 & 38.1 & 52.4 & 14.3 & 100.0 \\
\hline \multirow{2}{*}{ Control } & \multirow{2}{*}{ Nada } & $\mathrm{N} .^{\circ}$ & 8 & 11 & 15 & 9 & 9 & 26 \\
\hline & & $\%$ & 30.8 & 42.3 & 57.7 & 34.6 & 34.6 & 100.0 \\
\hline \multicolumn{9}{|c|}{ JUEGOS DE CONCENTRACIÓN } \\
\hline \multirow{2}{*}{$\begin{array}{l}\text { Experi- } \\
\text { mental }\end{array}$} & \multirow{2}{*}{$\begin{array}{l}\text { Total- } \\
\text { mente }\end{array}$} & $\mathrm{N} .^{\circ}$ & 2 & 7 & 4 & 5 & 0 & 9 \\
\hline & & $\%$ & 9.5 & 33.3 & 19.0 & 23.8 & 0.0 & 42.9 \\
\hline
\end{tabular}

\begin{tabular}{|c|c|c|c|c|c|c|c|c|}
\hline \multicolumn{9}{|c|}{ J1*\$COGNITIVAS*GRUPO tabulación cruzada* } \\
\hline \multirow{2}{*}{\multicolumn{2}{|c|}{ Grupo de estudio }} & & \multicolumn{5}{|c|}{ ESTRATEGIAS COGNITIVAS } & \multirow[t]{2}{*}{ Total } \\
\hline & & & MDA & $\mathrm{DA}$ & 1 & ED & MED & \\
\hline & \multirow{2}{*}{$\begin{array}{l}\text { Parcial- } \\
\text { mente }\end{array}$} & N. ${ }^{\circ}$ & 3 & 8 & 4 & 6 & 3 & 12 \\
\hline & & $\%$ & 14.3 & 38.1 & 19.0 & 28.6 & 14.3 & 57.1 \\
\hline & \multirow{2}{*}{ TOTAL } & N. ${ }^{\circ}$ & 5 & 15 & 8 & 11 & 3 & 21 \\
\hline & & $\%$ & $23.8 \%$ & 71.4 & 38.1 & 52.4 & 14.3 & 100.0 \\
\hline \multirow{2}{*}{ Control } & \multirow{2}{*}{ Nada } & $\mathrm{N} .^{\circ}$ & 8 & 11 & 15 & 9 & 9 & 26 \\
\hline & & $\%$ & 30.8 & 42.3 & 57.7 & 34.6 & 34.6 & 100.0 \\
\hline \multicolumn{9}{|c|}{ JUEGOS DE ESTRATEGIA } \\
\hline \multirow{6}{*}{$\begin{array}{l}\text { Experi- } \\
\text { mental }\end{array}$} & \multirow{2}{*}{$\begin{array}{l}\text { Total- } \\
\text { mente }\end{array}$} & N. ${ }^{\circ}$ & 4 & 6 & 5 & 5 & 2 & 11 \\
\hline & & $\%$ & 19.0 & 28.6 & 23.8 & 23.8 & 9.5 & 52.4 \\
\hline & \multirow{2}{*}{$\begin{array}{l}\text { Parcial- } \\
\text { mente }\end{array}$} & $N .^{\circ}$ & 1 & 9 & 3 & 6 & 1 & 10 \\
\hline & & $\%$ & 4.8 & 42.9 & 14.3 & 28.6 & 4.8 & 47.6 \\
\hline & \multirow{2}{*}{ TOTAL } & $\mathrm{N} .^{\circ}$ & 5 & 15 & 8 & 11 & 3 & 21 \\
\hline & & $\%$ & $23.8 \%$ & 71.4 & 38.1 & 52.4 & 14.3 & 100.0 \\
\hline \multirow{2}{*}{ Control } & \multirow{2}{*}{ Nada } & $\mathrm{N} .^{\circ}$ & 8 & 11 & 15 & 9 & 9 & 26 \\
\hline & & $\%$ & 30.8 & 42.3 & 57.7 & 34.6 & 34.6 & 100.0 \\
\hline \multicolumn{9}{|c|}{ JUEGOS DE LOGRO } \\
\hline \multirow{6}{*}{$\begin{array}{l}\text { Experi- } \\
\text { mental }\end{array}$} & \multirow{2}{*}{$\begin{array}{l}\text { Total- } \\
\text { mente }\end{array}$} & $\mathrm{N} .^{\circ}$ & 2 & 9 & 4 & 7 & 2 & 12 \\
\hline & & $\%$ & 9.5 & 42.9 & 19.0 & 33.3 & 9.5 & 57.1 \\
\hline & \multirow{2}{*}{$\begin{array}{l}\text { Parcial- } \\
\text { mente }\end{array}$} & $\mathrm{N} .^{\circ}$ & 3 & 6 & 4 & 4 & 1 & 9 \\
\hline & & $\%$ & 14.3 & 28.6 & 19.0 & 19.0 & 4.8 & 42.9 \\
\hline & \multirow{2}{*}{ TOTAL } & $\mathrm{N} .^{\circ}$ & 5 & 15 & 8 & 11 & 3 & 21 \\
\hline & & $\%$ & $23.8 \%$ & 71.4 & 38.1 & 52.4 & 14.3 & 100.0 \\
\hline \multirow{2}{*}{ Control } & \multirow{2}{*}{ Nada } & $\mathrm{N} .^{\circ}$ & 8 & 11 & 15 & 9 & 9 & 26 \\
\hline & & $\%$ & 30.8 & 42.3 & 57.7 & 34.6 & 34.6 & 100.0 \\
\hline
\end{tabular}

*Los porcentajes y los totales se basan en los encuestados Fuente: Evaluación general de los 2 grupos de estudio. Elaborado por: Ramírez \& Mayorga \& Lara, 2019

Se plantea una hipótesis de investigación: "el uso del interfaz gamificada potencia el uso de las estrategias de aprendizaje en los estudiantes de la asignatura de psicología social en la carrera de psicología de la UTI", para esto se usa estadística inferencial con SPSS versión 25 con prueba de T student para muestras independientes en donde se determina que se cuenta con medias diferentes para los grupos que utilizaron gamificación versus los que no; en la que se observa que la muestra tiene las medias pata en este estudio son significativas, como se muestra en la Tabla 5. 
Tabla 5

Estudio de medias independientes según t-student

\begin{tabular}{|c|c|c|c|c|c|}
\hline & $\begin{array}{l}\text { Uso } \\
\text { de la } \\
\text { Gamifi- } \\
\text { cación }\end{array}$ & $\mathrm{N}$ & $\begin{array}{l}\text { Me- } \\
\text { dia }\end{array}$ & $\begin{array}{l}\text { Des- } \\
\text { viación } \\
\text { están- } \\
\text { dar }\end{array}$ & $\begin{array}{l}\text { Media } \\
\text { de error } \\
\text { están- } \\
\text { dar }\end{array}$ \\
\hline \multirow{2}{*}{$\begin{array}{l}\text { Estrategia afectiva } \\
\text { - motivacionales }\end{array}$} & $\mathrm{Si}$ & 21 & 2.05 & 1.203 & .263 \\
\hline & No & 26 & 3.46 & 1.174 & .230 \\
\hline \multirow{2}{*}{$\begin{array}{l}\text { Estrategia afectiva } \\
\text { - afectivos }\end{array}$} & Si & 21 & 2.95 & 1.024 & .223 \\
\hline & No & 26 & 4.00 & .980 & .192 \\
\hline \multirow{2}{*}{$\begin{array}{l}\text { Estrategia afectiva } \\
\text { - meta cognitivas }\end{array}$} & $\mathrm{Si}$ & 21 & 1.81 & .873 & .190 \\
\hline & No & 26 & 2.31 & .970 & .190 \\
\hline \multirow{2}{*}{$\begin{array}{l}\text { Estrategia afectiva } \\
\text { - control y manejo } \\
\text { de recursos }\end{array}$} & $\mathrm{Si}$ & 21 & 2.19 & 1.123 & .245 \\
\hline & No & 26 & 3.12 & 1.336 & .262 \\
\hline \multirow{2}{*}{$\begin{array}{l}\text { Estrategia cognitiva } \\
\text { - búsqueda, reco- } \\
\text { gida y selección de } \\
\text { información }\end{array}$} & $\mathrm{Si}$ & 21 & 3.14 & .964 & .210 \\
\hline & No & 26 & 3.58 & 1.102 & .216 \\
\hline \multirow{2}{*}{$\begin{array}{l}\text { Estrategia cognitiva } \\
\text { - procesamiento y } \\
\text { uso de información }\end{array}$} & $\mathrm{Si}$ & 21 & 2.48 & 1.289 & .281 \\
\hline & No & 26 & 2.42 & 1.270 & .249 \\
\hline
\end{tabular}

Fuente: Evaluación general de los 2 grupos de estudio. Elaborado por: Ramírez \& Mayorga \& Lara, 2019
Fuente: Evaluación general de los 2 grupos de estudio. Elaborado por: Ramírez \& Mayorga \& Lara, 2019

El contraste de hipótesis se realizó usando la Prueba t de Student para muestras independientes, considerando un nivel de significancia de 0.05 ( $5 \%$ de error). Debido a ello: Sí p $>0.05$, se concluye con la hipótesis nula (H0) Sí p $<0.05$, se concluye con la hipótesis alterna (H1).

La prueba de Levene demuestra que para el caso de las estrategias afectivas emocionales y cognitiva de procesamiento y uso de información al presentar un valor inferior a 0.05 las varianzas son iguales mientras que para el resto de estrategias las varianzas son desiguales. Se observa el estadístico t con su nivel de significación bilateral, este valor nos informa sobre el grado de compatibilidad entre la hipótesis de igualdad de medias y las diferencia entre medias poblacionales observadas; en nuestro caso es mayor que 0.05 , la conclusión es que hay compatibilidad entre la hipótesis de igualdad de medias poblacionales y las diferencias entre las medias de grupos representados por gru-

Tabla 6

Prueba de muestras independientes

\begin{tabular}{|c|c|c|c|c|c|c|c|c|c|c|}
\hline & & \multicolumn{2}{|c|}{$\begin{array}{l}\text { Prueba de } \\
\text { Levene de } \\
\text { calidad de } \\
\text { varianzas }\end{array}$} & \multicolumn{7}{|c|}{ Prueba t para la igualdad de medias } \\
\hline & & \multirow{2}{*}{$\mathrm{F}$} & \multirow{2}{*}{ Sig. } & \multirow{2}{*}{$t$} & \multirow{2}{*}{$\mathrm{gl}$} & \multirow{2}{*}{$\begin{array}{l}\text { Sig. } \\
\text { (bila- } \\
\text { teral) }\end{array}$} & \multirow{2}{*}{$\begin{array}{l}\text { Dif. de } \\
\text { me- } \\
\text { dias }\end{array}$} & \multirow{2}{*}{$\begin{array}{l}\text { Dif. de } \\
\text { error } \\
\text { están- } \\
\text { dar }\end{array}$} & \multicolumn{2}{|c|}{$\begin{array}{l}95 \% \text { intérvalo de con- } \\
\text { fianza de la diferencia }\end{array}$} \\
\hline & & & & & & & & & Inferior & Superior \\
\hline \multirow{2}{*}{$\begin{array}{l}\text { Estrategia afectiva } \\
\text { - motivacionales }\end{array}$} & Se asume varianzas iguales & .038 & .846 & -4.060 & 45 & .000 & -1.414 & .348 & -2.115 & -.712 \\
\hline & No se asume varianzas iguales & & & -4.049 & 42.488 & .000 & -1.414 & .349 & -2.118 & -.709 \\
\hline \multirow{2}{*}{$\begin{array}{l}\text { Estrategia afectiva } \\
\text { - afectivos }\end{array}$} & Se asume varianzas iguales & .074 & .786 & -3.573 & 45 & .001 & -1.048 & .293 & -1.638 & -.457 \\
\hline & No se asume varianzas iguales & & & -3.556 & 42.108 & .001 & -1.048 & .295 & -1.642 & -.453 \\
\hline \multirow{2}{*}{$\begin{array}{l}\text { Estrategia afectiva } \\
\text { - meta cognitivas }\end{array}$} & Se asume varianzas iguales & .416 & .522 & -1.829 & 45 & .074 & -.498 & .272 & -1.047 & .050 \\
\hline & No se asume varianzas iguales & & & -1.850 & 44.435 & .071 & -.498 & .269 & -1.041 & .044 \\
\hline \multirow{2}{*}{$\begin{array}{l}\text { Estrategia afectiva } \\
\text { - control y manejo } \\
\text { de recursos }\end{array}$} & Se asume varianzas iguales & 1.290 & .262 & -2.530 & 45 & .015 & -.925 & .366 & -1.661 & -.188 \\
\hline & No se asume varianzas iguales & & & -2.577 & 44.911 & .013 & -.925 & .359 & -1.648 & -.202 \\
\hline \multirow{2}{*}{$\begin{array}{l}\text { Estrategia cognitiva } \\
\text { - búsqueda, reco- } \\
\text { gida y selección de } \\
\text { información }\end{array}$} & Se asume varianzas iguales & .869 & .356 & -1.419 & 45 & .163 & -.434 & .306 & -1.050 & .182 \\
\hline & No se asume varianzas iguales & & & -1.440 & 44.681 & .157 & -.434 & .302 & -1.041 & .173 \\
\hline \multirow{2}{*}{$\begin{array}{l}\text { Estrategia cognitiva } \\
\text { - procesamiento y } \\
\text { uso de información }\end{array}$} & Se asume varianzas iguales & .001 & .979 & .142 & 45 & .888 & .053 & .375 & -.703 & .809 \\
\hline & No se asume varianzas iguales & & & .141 & 42.675 & .888 & .053 & .376 & -.705 & .811 \\
\hline
\end{tabular}


po que utilizó la gamificación y el grupo que no lo hizo para los datos del pretest y posttest.

Para el caso de las estrategias afectivas con el resultado de t student obtenido y un valor de significancia menor a 0.05 , se determina que la implementación de una interfaz gamificada en la asignatura de psicología social influye significativamente en el uso de las estrategias de aprendizaje AFECTIVAS más no en las COGNITIVAS en los estudiantes de la Universidad Tecnológica Indoamérica (UTI).

\section{Discusión y Conclusiones}

La aplicación del interfaz con la metodología de gamificación potencia el aprendizaje de una manera amigable y bidireccional, porque ayuda al usuario en la motivación, la concentración, el compromiso, el esfuerzo y la fidelización, entre otros valores, la Prueba de T student demuestra un valor de significancia menor a 0.05 , se determina que la implementación de una interfaz gamificada en la asignatura de psicología social influye significativamente en el uso de las estrategias de aprendizaje

La base de datos MYSQL es adaptable a cualquier materia lo que contribuye a mejorar y retroalimentar oportunamente el proceso enseñanza - aprendizaje ya que la misma es inmediata, a la vez el rendimiento escolar también incide en un proceso de mejora, el docente es el administrador y puede gestionar a través de los juegos actividades que potencian estrategias de aprendizaje en el proceso de estudio de la asignatura La interfaz gamificada permitió desarrollar componentes motivacionales en el proceso de aprendizaje, se reduce el nivel de estrés que representan los reactivos de aprendizaje en los estudiantes a través del juego y se incide positivamente en el rendimiento académico.

Se demostró que las estrategias de aprendizaje son un elemento fundamental en el aprendizaje y contribuye al desarrollo de habilidades y competencias de los estudiantes de la carrera de sicología, específicamente en la asignatura de sicología social. Se corroboró que la aplicación de estrategias de aprendizaje apoyadas en la gamificación, incidieron de manera significativa en el rendimiento aca- démico de los estudiantes que se tomaron como grupo experimental, a diferencia de que a quienes no se les aplicó la experimentación tienen un nivel de rendimiento inferior, el desempeño académico se elevó en una relación de 2 a 1 . Para la validación de hipótesis se utilizó la prueba estadística de T student para muestras independientes, en donde se asumen varianzas iguales para el caso de del uso de la gamificación y las estrategias de aprendizaje afectivas, situación que no se presenta en las estrategias de aprendizaje cognitivas, lo que quienes utilizaron la técnica del juego a través de la plataforma gamificada tuvieron un mejor desempeño, influyendo de manera significativa en las estrategias de aprendizaje afectivas. Un aspecto fundamental en el desarrollo de la presente investigación es la planificación docente ya que el tratamiento del contenido, las actividades, el material determinan el desarrollo de habilidades que responden al perfil de egreso del estudiante y al desarrollo de competencias.

En relación con la planificación del docente y en función de la temática a tratar, se destaca que el juego es un elemento fundamental en el proceso de aprendizaje, el cual fue gestionado en la plataforma gamificada y se convierte en un componente motivacional, subyuga el nivel de tensión en la evaluación convirtiendo al proceso en amigable y bidireccional, presenta una retroalimentación inmediata al estudiante; respecto a la planificación la investigación se relaciona por lo expresado por Contreras, Masa \& Melo (2017), respecto al "Uso del modelo de aprendizaje inverso para mejorar materiales educativos universitarios" y publicada en la Revista Ibérica de Sistemas e Tecnologías de Información donde se destaca el estudio experimental de seguimiento de algunos de los materiales utilizados en la docencia universitaria para comprobar si cumplen la función formativa que había sido prevista, y, en el caso de que se detecte que no es así, realizar una mejora en los mismos para que cumplan su función (Contreras, Arias, \& Melo, 2017). Para el presente estudio el uso de ésta herramienta tecnológica permitió de forma rápida, dinámica y sostenida la implementación de estrategias de aprendizaje que creemos también se irradiaron en el aprendizaje de otras asignaturas, las cuales sería conveniente investigar en qué medida fueron impactadas por el presente estudio. 
Otro elemento que se destaca es el valorar de lo planificado en la gestión de actividades versus los resultados alcanzados en la ejecución de los juegos ya que estos buscan la comprensión, inducción-deducción, el análisis y la reflexión que son competencias propias del perfil de egreso del estudiante de la carrera de psicología de la UTI.

La evaluación medida por el juego se convierte en un elemento motivacional y pierde el peso de estrés que normalmente se refleja en los estudiantes, al procesar la información de las respuestas en una plataforma el profesor puede conocer de forma específica los contenidos fáciles y difíciles de comprender por el estudiante, para luego darles un tratamiento y retroinformación formativa en el taller desarrollado en clase (Prieto \& Díaz, 2018).

\section{REFERENCIAS BIBLIOGRÁFICAS}

Acedo, M. (2013). 10 Pros And Cons Of A Flipped Classroom. Recuperado de http://www.teachthought.com/learning/blended-flipped-learning/10-pros-cons-flippedclassroom/

Aguerrondo, I. (2017). El nuevo paradigma de la educación para el siglo XXI. OEI. Programas. Desarrollo Escolar y Administración Educativa. Recuperado de http:// beu.extension.unicen.edu.ar/xmlui/bitstream/handle/123456789/58/El\%20Nuevo\%20Paradigma\%20de\%20 la $\% 20$ Educaci $\%$ C3\%B3n $\% 20$ para $\% 20$ el $\% 20$ siglo $\% 20$ XXI.pdf?sequence $=1$

Ángel C., J., Arias M., J., Melo A., M. G., \& Martín E., R. (2017). Uso del modelo de aprendizaje inverso para mejorar materiales educativos universitarios. RISTI-Revista Ibérica de Sistemas e Tecnologias de Informação, (23), 17-32. https://doi.org/10.17013/risti.23.17-32

Arteaga-Paz, L. G., \& Basurto-Vera, P. R. (2017). Una aproximación teórico conceptual a la tecnología educativa. Dominio de las Ciencias, 3(3), 657-675.

Berenguer-Albaladejo, C. B. (2016). Acerca de la utilidad del aula invertida o flipped classroom. En: XIV Jornadas de Redes de Investigación en Docencia Universitaria: Investigación, innovación y enseñanza universitaria: enfoques pluridisciplinares, 1466-1480. Instituto de Ciencias de la Educación. Recuperado de http://rua.ua.es/dspace/handle/10045/59358

Bergmann, J., \& Sams, A. (2012). Flip your classroom: Reach every student in every class every day. International society for technology in education.

Cabero A., J. (2017). La formación en la era digital: ambientes enriquecidos por la tecnología. Revista Gestión de la
Innovación en Educación Superior, 2(2), 41-64. Recuperado de http://ojs.inacap.cl/index.php/regies/article/view/24

Cobo, A., Gómez, P., Pérez, D., \& Rocha, R. (2005). PHP y MySQL. Tecnología de desarrollo y aplicaciones web. Espańa: Ediciones Díaz de Santos.

Contreras, Juan Ángel, Masa, Juan Arias, Andrade, Malena G. Melo, \& Espada, Rafael Martín. (2017). Uso del modelo de aprendizaje inverso para mejorar materiales educativos universitarios. RISTI - Revista Ibérica de Sistemas e Tecnologias de Informação, (23), 17-32. https://dx.doi. org/10.17013/risti.23.17-32

Díaz Cruzado, J., \& Troyano Rodríguez, Y. (2013). El potencial de la gamificación aplicado al ámbito educativo. III Jornadas de Innovación Docente. Innovación Educativa: respuesta en tiempos de incertidumbre. Recuperado de https://idus.us.es/xmlui/handle/11441/59067

Esteban, A. M., \& Zapata, R. M. (2016). Estrategias de aprendizaje y eLearning. Un apunte para la fundamentación del diseño educativo en los entornos virtuales de aprendizaje. Revista de Educación a Distancia.

Gaitán, V. (1 de noviembre de 2013). Gamificación: el aprendizaje divertido. Recuperado el 14 de diciembre de 2019, de https://www.educativa.com/blog-articulos/gamificacion-el-aprendizaje-divertido/

Gallego, F. J., Villagrá, C. J., Satorre, R., Compañ, P., Molina, R., \& Llorens Largo, F. (2014). Panorámica: serious games, gamification y mucho más. ReVisión, 7(2). Recuperado de http://aenui.net/ojs/index.php?journal=revision\&page $=$ article \&op=viewArticle \& path $\% 5 B \% 5 D=148 \&$ path $\%$ $5 \mathrm{~B} \% 5 \mathrm{D}=238$

Gargallo, B., Almerich, G.; Suárez-Rodríguez, J. M. \& García-Félix, E. (2012). Estrategias de aprendizaje en estudiantes universitarios excelentes y medios. Su evolución a lo largo del primer año de carrera. RELIEVE, 18(2). https://doi. org/10.7203/relieve.18.2.2000

Gargallo, B., Suárez-Rodríguez, J. M., \& Pérez-Pérez, C. (2009). El cuestionario CEVEAPEU. Un instrumento para la evaluación de las estrategias de aprendizaje de los estudiantes universitarios. Relieve, 15(2). https://doi.org/10.7203/ relieve.15.2.4156

Hernández, R., Fernández, C. \& Baptista, M. (2014). Metodología de la Investigación (5a ed.). México: Mc. Graw-Hill.

Hernández, R., Fernández, C., \& Baptista, P. (1996). Metodología de la investigación. Edici6n McGraw-Hill, 1.

INEC. (2015). Encuesta Nacional de Empleo Desempleo y Subempleo - ENEMDU. Quito - Ecuador: INEC.

Landers, R. N., \& Callan, Rachel C. Casual social games as serious games: The psychology of gamification in undergraduate education and employee training. En: Ma, M.; Oikonomou, A.; Jain, L. (Ed.). Serious games and edutainment applications. Londres: Springer, 2011. p. 399-423. https:// doi.org/10.1007/978-1-4471-2161-9_20

Martínez-Olvera, W., Esquivel-Gámez, I., \& Martínez C., J. (2014). Aula Invertida o Modelo Invertido de Aprendiza- 
je: origen, sustento e implicaciones. Recuperado de https:// www. researchgate. net/publication/273765424_Aula_Invertida_o_Modelo_Invertido_de_Aprendizaje_origen_sustento_e_implicaciones.

Pintrich, P. R., Smith, D. A. F. García, T. y Mackeachie, W.J. (1991). A manual for the use of the Motivated Strategies for Learning Questionnaire (MSLQ). Ann Arbor: Universidad de Michigan. Technical Report No. 91-B-004.

Prieto, M., A., Díaz M., D., Lara A., I., \& Monserrat S., J. (2018). Nuevas combinaciones de aula inversa con just in time teaching y análisis de respuestas de alumnos. RIED. Revista Iberoamericana de Educación a Distancia, 21(1), 175-194. https://doi.org/10.5944/ried.21.1.18836

Requena, B. E. S. (2016). Las TIC y la educación social en el siglo XXI. EDMETIC, 5(1), 8-24. https://doi. org/10.21071/edmetic.v5i1.4014

Revelo-Sánchez, O., Collazos-Ordónez, C. A., \& Jiménez-Toledo, J. A. (2018). El trabajo colaborativo como estrategia didáctica para la enseñanza/aprendizaje de la programación: una revisión sistemática de literatura. TecnoLógicas, 21(41), 115-134. https://doi.org/10.22430/22565337.731

Rivera C., F. M., \& García M., A. (2018). Aula invertida con tecnologías emergentes en ambientes virtuales en la Universidad Politécnica Salesiana del Ecuador. Revista Cubana de Educación Superior, 37(1), 108-123. Recuperado de http://scielo.sld.cu/scielo.php?script=sci_arttext\&pi$\mathrm{d}=$ S0257-43142018000100008

Rodríguez, M. E. (2017). Currículum, educación y cultura en la formación docente del siglo XXI desde la complejidad. Educación y Humanismo, 19(33), 425-440. https://doi. org/10.17081/eduhum.19.33.2654

Sánchez i Peris, F. J. (2015). Gamificación. Education in the Knowledge Society, 16(2), 13-15. https://doi.org/10.14201/ eks20151621315

Sánchez, L. P. (2014). Estrategias de aprendizaje. Función y diagnóstico en el aprendizaje adolescente. Padres y Maestros/Journal of Parents and Teachers, 36.

Teixes, F. (2015). Gamificación: fundamentos y aplicaciones. UOC.

Welling, L., \& Thomson, L. (2003). Desarrollo web con PHP y MYSQL. Madrid, Espańa: Editorial ANAYA.

Weinstein, C. E., Palmer, D. R. y Schulte, A.C. (1987). LASSI: Learning and Study Strategies Inventory. Clearwater, FL: Publishing Company 
Anexo

\section{ANEXO 1. Cuestionario de Gargallo}

Universidad:

Facultad o Escuela:

Título universitario que se está estudiando:

Sexo: Hombre $\square \quad$ Mujer

Ciclo: Primer ciclo $\square \quad$ Segundo ciclo

Curso que se está realizando: Primero $\square \quad$ Segundo $\square$ Tercero $\square \quad$ Cuarto $\square$ Quinto

Edad: 17-18 $\square \quad$ 19-20 $\square \quad 21-22 \square \quad 23-24 \square \quad 25-26 \square \quad 27-28 \square \quad$ más de $28 \square$

Elección de la carrera: primera opción $\square \quad$ segunda opción $\square \quad$ tercera opción $\square$ cuarta opción en otras opción

Nivel de estudios de los padres:

Del padre

De la madre

Sin estudios

Sin estudios

Estudios primarios

Estudios primarios

Estudios secundarios

Estudios secundarios

Bachillerato

Bachillerato

Universitarios medios

Universitarios medios

Universitarios superiores

Universitarios superiores

Doctor

Doctor

Calificaciones de las asignaturas del curso anterior:

\begin{tabular}{|c|c|c|c|c|c|}
\hline & Suspenso & Aprobado & Notable & sobresaliente & M.Honor \\
\hline & Suspenso & Aprobado & Notable & sobresaliente & M.Honor \\
\hline & - Suspenso & Aprobado & Notable & sobresaliente & M.Hone \\
\hline 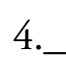 & Suspenso & Aprobado & Notable & sobresaliente & M.Honor \\
\hline- & Suspenso & Aprobado & Notable & sobresaliente & M.Hon \\
\hline$r$ & Suspenso & Aprobado & Notable & sobresaliente & M.Hon \\
\hline & Suspenso & Aprobado & Notable & sobresaliente & IVI. Honor \\
\hline & Suspenso & Aprobado & Notable & sobresaliente & M.H \\
\hline
\end{tabular}




\section{B) Respuestas a los ítems del cuestionario:}

\begin{tabular}{|c|c|c|c|c|c|}
\hline & $\begin{array}{c}\text { Muy en } \\
\text { desacuerdo }\end{array}$ & $\begin{array}{l}\text { En des- } \\
\text { acuerdo }\end{array}$ & Indeciso & $\begin{array}{c}\text { De } \\
\text { acuerdo }\end{array}$ & $\begin{array}{l}\text { Muy de } \\
\text { acuerdo }\end{array}$ \\
\hline $\begin{array}{l}\text { 1. Lo que más me satisface es entender los contenidos } \\
\text { a fondo }\end{array}$ & & & & & \\
\hline $\begin{array}{l}\text { 2. Aprender de verdad es lo más importante para mí en } \\
\text { la universidad }\end{array}$ & & & & & \\
\hline 3. Cuando estudio lo hago con interés por aprender & & & & & \\
\hline $\begin{array}{l}\text { 4. Estudio para no defraudar a mi familia y a la gente que } \\
\text { me importa }\end{array}$ & & & & & \\
\hline $\begin{array}{l}\text { 5. Necesito que otras personas -padres, amigos, profeso- } \\
\text { res, etc.- me animen para estudiar }\end{array}$ & & & & & \\
\hline $\begin{array}{l}\text { 6. Lo que aprenda en unas asignaturas lo podré utilizar en } \\
\text { otras y también en mi futuro profesional }\end{array}$ & & & & & \\
\hline $\begin{array}{l}\text { 7. Es importante que aprenda las asignaturas por el valor } \\
\text { que tienen para mi formación }\end{array}$ & & & & & \\
\hline $\begin{array}{l}\text { 8. Creo que es útil para mí aprenderme las asignaturas } \\
\text { de este curso }\end{array}$ & & & & & \\
\hline $\begin{array}{l}\text { 9. Considero muy importante entender los contenidos de } \\
\text { las asignaturas }\end{array}$ & & & & & \\
\hline 10. Mi rendimiento académico depende de mi esfuerzo & & & & & \\
\hline 11. Mi rendimiento académico depende de mi capacidad & & & & & \\
\hline 12. Mi rendimiento académico depende de la suerte & & & & & \\
\hline 13. Mi rendimiento académico depende de los profesores & & & & & \\
\hline $\begin{array}{l}\text { 14. Mi rendimiento académico depende de mi habilidad } \\
\text { para organizarme }\end{array}$ & & & & & \\
\hline $\begin{array}{l}\text { 15. Estoy seguro de que puedo entender incluso los con- } \\
\text { tenidos más difíciles de las asignaturas de este curso }\end{array}$ & & & & & \\
\hline $\begin{array}{l}\text { 16. Puedo aprenderme los conceptos básicos que se en- } \\
\text { señan en las diferentes materias }\end{array}$ & & & & & \\
\hline $\begin{array}{l}\text { 17. Soy capaz de conseguir en estos estudios lo que me } \\
\text { proponga }\end{array}$ & & & & & \\
\hline $\begin{array}{l}\text { 18. Estoy convencido de que puedo dominar las habilida- } \\
\text { des que se enseñan en las diferentes asignaturas }\end{array}$ & & & & & \\
\hline $\begin{array}{l}\text { 19. La inteligencia supone un conjunto de habilidades que } \\
\text { se puede modificar e incrementar con el propio esfuerzo } \\
\text { y el aprendizaje }\end{array}$ & & & & & \\
\hline $\begin{array}{l}\text { 20. La inteligencia se tiene o no se tiene y no se puede } \\
\text { mejorar }\end{array}$ & & & & & \\
\hline 21. Normalmente me encuentro bien físicamente & & & & & \\
\hline 22. Duermo y descanso lo necesario & & & & & \\
\hline $\begin{array}{l}\text { 23. Habitualmente mi estado anímico es positivo y me } \\
\text { siento bien }\end{array}$ & & & & & \\
\hline 24. Mantengo un estado de ánimo apropiado para trabajar & & & & & \\
\hline 25. Cuando hago un examen, me pongo muy nervioso & & & & & \\
\hline $\begin{array}{l}\text { 26. Cuando he de hablar en público me pongo muy ner- } \\
\text { vioso }\end{array}$ & & & & & \\
\hline $\begin{array}{l}\text { 27. Mientras hago un examen, pienso en las consecuen- } \\
\text { cias que tendría suspender }\end{array}$ & & & & & \\
\hline
\end{tabular}




\begin{tabular}{|c|c|c|c|c|c|}
\hline & $\begin{array}{c}\text { Muy en } \\
\text { desacuerdo }\end{array}$ & $\begin{array}{l}\text { En des- } \\
\text { acuerdo }\end{array}$ & Indeciso & $\begin{array}{l}\text { De } \\
\text { acuerdo }\end{array}$ & $\begin{array}{l}\text { Muy de } \\
\text { acuerdo }\end{array}$ \\
\hline $\begin{array}{l}\text { 28. Soy capaz de relajarme y estar tranquilo en situacio- } \\
\text { nes de estrés como exámenes, exposiciones o interven- } \\
\text { ciones en público }\end{array}$ & & & & & \\
\hline $\begin{array}{l}\text { 29. Sé cuáles son mis puntos fuertes y mis puntos débiles, } \\
\text { al enfrentarme al aprendizaje de las asignaturas }\end{array}$ & & & & & \\
\hline $\begin{array}{l}\text { 30. Conozco los criterios de evaluación con los que me } \\
\text { van a evaluar los profesores en las diferentes materias }\end{array}$ & & & & & \\
\hline 31. Sé cuáles son los objetivos de las asignaturas & & & & & \\
\hline $\begin{array}{l}\text { 32. Planifico mi tiempo para trabajar las asignaturas a lo } \\
\text { largo del curso }\end{array}$ & & & & & \\
\hline $\begin{array}{l}\text { 33. Llevo al día el estudio de los temas de las diferentes } \\
\text { asignaturas }\end{array}$ & & & & & \\
\hline 34. Sólo estudio antes de los exámenes & & & & & \\
\hline $\begin{array}{l}\text { 35. Tengo un horario de trabajo personal y estudio, al mar- } \\
\text { gen de las clases }\end{array}$ & & & & & \\
\hline $\begin{array}{l}\text { 36. Me doy cuenta de cuándo hago bien las cosas -en las } \\
\text { tareas académicas- sin necesidad de esperar la califica- } \\
\text { ción del profesor }\end{array}$ & & & & & \\
\hline $\begin{array}{l}\text { 37. Cuando veo que mis planes iniciales no logran el éxi- } \\
\text { to esperado, en los estudios, los cambio por otros más } \\
\text { adecuados }\end{array}$ & & & & & \\
\hline $\begin{array}{l}\text { 38. Si es necesario, adapto mi modo de trabajar a las exi- } \\
\text { gencias de los diferentes profesores y materias }\end{array}$ & & & & & \\
\hline $\begin{array}{l}\text { 39. Cuando he hecho un examen, sé si está mal o si está } \\
\text { bien }\end{array}$ & & & & & \\
\hline $\begin{array}{l}\text { 40. Dedico más tiempo y esfuerzo a las asignaturas difí- } \\
\text { ciles }\end{array}$ & & & & & \\
\hline $\begin{array}{l}\text { 41. Procuro aprender nuevas técnicas, habilidades y pro- } \\
\text { cedimientos para estudiar mejor y rendir más }\end{array}$ & & & & & \\
\hline $\begin{array}{l}\text { 42. Si me ha ido mal en un examen por no haberlo es- } \\
\text { tudiado bien, procuro aprender de mis errores y estudiar } \\
\text { mejor la próxima vez }\end{array}$ & & & & & \\
\hline $\begin{array}{l}\text { 43. Cuando me han puesto una mala calificación en un } \\
\text { trabajo, hago lo posible para descubrir lo que era incorrec- } \\
\text { to y mejorar en la próxima ocasión }\end{array}$ & & & & & \\
\hline $\begin{array}{l}\text { 44. Trabajo y estudio en un lugar adecuado -luz, tempe- } \\
\text { ratura, ventilación, ruidos, materiales necesarios a mano, } \\
\text { etc.- }\end{array}$ & & & & & \\
\hline $\begin{array}{l}\text { 45. Normalmente estudio en un sitio en el que pueda con- } \\
\text { centrarme en el trabajo }\end{array}$ & & & & & \\
\hline 46. Aprovecho bien el tiempo que empleo en estudiar & & & & & \\
\hline 47. Creo un ambiente de estudio adecuado para rendir & & & & & \\
\hline $\begin{array}{l}\text { 48. Procuro estudiar o realizar los trabajos de clase con } \\
\text { otros compañeros }\end{array}$ & & & & & \\
\hline $\begin{array}{l}\text { 49. Suelo comentar dudas relativas a los contenidos de } \\
\text { clase con los compañeros }\end{array}$ & & & & & \\
\hline $\begin{array}{l}\text { 50. Escojo compañeros adecuados para el trabajo en } \\
\text { equipo }\end{array}$ & & & & & \\
\hline 51. Me llevo bien con mis compañeros de clase & & & & & \\
\hline
\end{tabular}




\begin{tabular}{|c|c|c|c|c|c|}
\hline & $\begin{array}{c}\text { Muy en } \\
\text { desacuerdo }\end{array}$ & $\begin{array}{l}\text { En des- } \\
\text { acuerdo }\end{array}$ & Indeciso & $\begin{array}{c}\text { De } \\
\text { acuerdo }\end{array}$ & $\begin{array}{l}\text { Muy de } \\
\text { acuerdo }\end{array}$ \\
\hline \multicolumn{6}{|l|}{ 52. El trabajo en equipo me estimula a seguir adelante } \\
\hline \multicolumn{6}{|l|}{$\begin{array}{l}\text { 53. Cuando no entiendo algún contenido de una asignatu- } \\
\text { ra, pido ayuda a otro compañero }\end{array}$} \\
\hline \multicolumn{6}{|l|}{$\begin{array}{l}\text { 54. Conozco dónde se pueden conseguir los materiales } \\
\text { necesarios para estudiar las asignaturas }\end{array}$} \\
\hline \multicolumn{6}{|l|}{$\begin{array}{l}\text { 55. Me manejo con habilidad en la biblioteca y sé encon- } \\
\text { trar las obras que necesito }\end{array}$} \\
\hline \multicolumn{6}{|l|}{$\begin{array}{l}\text { 56. Sé utilizar la hemeroteca y encontrar los artículos que } \\
\text { necesito }\end{array}$} \\
\hline \multicolumn{6}{|l|}{$\begin{array}{l}\text { 57. No me conformo con el manual y/o con los apuntes } \\
\text { de clase, busco y recojo más información para las asig- } \\
\text { naturas }\end{array}$} \\
\hline \multicolumn{6}{|l|}{$\begin{array}{l}\text { 58. Soy capaz de seleccionar la información necesaria } \\
\text { para estudiar con garantías las asignaturas }\end{array}$} \\
\hline \multicolumn{6}{|l|}{$\begin{array}{l}\text { 59. Selecciono la información que debo trabajar en las } \\
\text { asignaturas pero no tengo muy claro si lo que yo seleccio- } \\
\text { no es lo correcto para tener buenas calificaciones }\end{array}$} \\
\hline \multicolumn{6}{|l|}{$\begin{array}{l}\text { 60. Soy capaz de separar la información fundamental de } \\
\text { la que no lo es para preparar las asignaturas }\end{array}$} \\
\hline \multicolumn{6}{|l|}{$\begin{array}{l}\text { 61. Cuando hago búsquedas en Internet, donde hay tan- } \\
\text { tos materiales, soy capaz de reconocer los documentos } \\
\text { que son fundamentales para lo que estoy trabajando o } \\
\text { estudiando }\end{array}$} \\
\hline \multicolumn{6}{|l|}{$\begin{array}{l}\text { 62. Cuando estudio los temas de las asignaturas, realizo } \\
\text { una primera lectura que me permita hacerme una idea de } \\
\text { lo fundamental }\end{array}$} \\
\hline \multicolumn{6}{|l|}{$\begin{array}{l}\text { 63. Antes de memorizar las cosas leo despacio para com- } \\
\text { prender a fondo el contenido }\end{array}$} \\
\hline \multicolumn{6}{|l|}{$\begin{array}{l}\text { 64. Cuando no comprendo algo lo leo de nuevo hasta que } \\
\text { me aclaro }\end{array}$} \\
\hline \multicolumn{6}{|l|}{$\begin{array}{l}\text { 65. Tomo apuntes en clase y soy capaz de recoger la in- } \\
\text { formación que proporciona el profesor } \\
\end{array}$} \\
\hline \multicolumn{6}{|l|}{$\begin{array}{l}\text { 66. Cuando estudio, integro información de diferentes } \\
\text { fuentes: clase, lecturas, trabajos prácticos, etc. }\end{array}$} \\
\hline \multicolumn{6}{|l|}{$\begin{array}{l}\text { 67. Amplío el material dado en clase con otros libros, re- } \\
\text { vistas, artículos, etc. }\end{array}$} \\
\hline \multicolumn{6}{|l|}{$\begin{array}{l}\text { 68. Trato de entender el contenido de las asignaturas es- } \\
\text { tableciendo relaciones entre los libros o lecturas recomen- } \\
\text { dadas y los conceptos expuestos en clase }\end{array}$} \\
\hline \multicolumn{6}{|l|}{$\begin{array}{l}\text { 69. Hago gráficos sencillos, esquemas o tablas para orga- } \\
\text { nizar la materia de estudio }\end{array}$} \\
\hline \multicolumn{6}{|l|}{$\begin{array}{l}\text { 70. Hago esquemas con las ideas importantes de los te- } \\
\text { mas }\end{array}$} \\
\hline \multicolumn{6}{|l|}{ 71. Hago resúmenes del material que tengo que estudiar } \\
\hline \multicolumn{6}{|l|}{$\begin{array}{l}\text { 72. Para estudiar selecciono los conceptos clave del tema } \\
\text { y los uno o relaciono mediante mapas conceptuales u } \\
\text { otros procedimientos }\end{array}$} \\
\hline $\begin{array}{l}\text { 73. Analizo críticamente los conceptos y las teorías que } \\
\text { me presentan los profesores }\end{array}$ & & & & & \\
\hline
\end{tabular}




\begin{tabular}{|c|c|c|c|c|c|}
\hline & $\begin{array}{c}\text { Muy en } \\
\text { desacuerdo }\end{array}$ & $\begin{array}{l}\text { En des- } \\
\text { acuerdo }\end{array}$ & Indeciso & $\begin{array}{c}\text { De } \\
\text { acuerdo }\end{array}$ & $\begin{array}{l}\text { Muy de } \\
\text { acuerdo }\end{array}$ \\
\hline \multicolumn{6}{|l|}{$\begin{array}{l}\text { 74. En determinados temas, una vez que los he estudiado } \\
\text { y he profundizado en ellos, soy capaz de aportar ideas } \\
\text { personales y justificarlas }\end{array}$} \\
\hline \multicolumn{6}{|l|}{$\begin{array}{l}\text { 75. Me hago preguntas sobre las cosas que oigo, leo y } \\
\text { estudio, para ver si las encuentro convincentes }\end{array}$} \\
\hline \multicolumn{6}{|l|}{$\begin{array}{l}\text { 76. Cuando en clase o en los libros se expone una teoría, } \\
\text { interpretación o conclusión, trato de ver si hay buenos ar- } \\
\text { gumentos que la sustenten }\end{array}$} \\
\hline \multicolumn{6}{|l|}{$\begin{array}{l}\text { 77. Cuando oigo o leo una afirmación, pienso en otras al- } \\
\text { ternativas posibles }\end{array}$} \\
\hline \multicolumn{6}{|l|}{$\begin{array}{l}\text { 78. Para aprender las cosas, me limito a repetirlas una y } \\
\text { otra vez }\end{array}$} \\
\hline \multicolumn{6}{|l|}{$\begin{array}{l}\text { 79. Me aprendo las cosas de memoria, aunque no las } \\
\text { comprenda }\end{array}$} \\
\hline \multicolumn{6}{|l|}{$\begin{array}{l}\text { 80. Cuando he de aprender cosas de memoria (listas de } \\
\text { palabras, nombres, fechas...), las organizo según algún } \\
\text { criterio para aprenderlas con más facilidad (por ejemplo, } \\
\text { familias de palabras) }\end{array}$} \\
\hline \multicolumn{6}{|l|}{$\begin{array}{l}\text { 81. Para recordar lo estudiado me ayudo de esquemas } \\
\text { o resúmenes hechos con mis palabras que me ayudan a } \\
\text { retener mejor los contenidos }\end{array}$} \\
\hline \multicolumn{6}{|l|}{$\begin{array}{l}\text { 82. Para memorizar utilizo recursos mnemotécnicos tales } \\
\text { como acrónimos (hago una palabra con las primeras le- } \\
\text { tras de varios apartados que debo aprender), siglas, pa- } \\
\text { labras clave, etc. }\end{array}$} \\
\hline \multicolumn{6}{|l|}{$\begin{array}{l}\text { 83. Hago uso de palabras clave que estudié y aprendí, } \\
\text { para recordar los contenidos relacionados con ellas }\end{array}$} \\
\hline \multicolumn{6}{|l|}{$\begin{array}{l}\text { 84. Antes de empezar a hablar o escribir, pienso y preparo } \\
\text { mentalmente lo que voy a decir o escribir }\end{array}$} \\
\hline \multicolumn{6}{|l|}{$\begin{array}{l}\text { 85. A la hora de responder un examen, antes de redactar, } \\
\text { recuerdo todo lo que puedo, luego lo ordeno o hago un } \\
\text { esquema o guion y finalmente lo desarrollo }\end{array}$} \\
\hline \multicolumn{6}{|l|}{$\begin{array}{l}\text { 86. Utilizo lo aprendido en la universidad en las situacio- } \\
\text { nes de la vida cotidiana }\end{array}$} \\
\hline \multicolumn{6}{|l|}{$\begin{array}{l}\text { 87. En la medida de lo posible, utilizo la aprendido en una } \\
\text { asignatura también en otras }\end{array}$} \\
\hline $\begin{array}{l}\text { 88. Cuando tengo que afrontar tareas nuevas, recuerdo lo } \\
\text { que ya sé y he experimentado para aplicarlo, si puedo, a } \\
\text { esa nueva situación }\end{array}$ & & & & & \\
\hline
\end{tabular}

